\title{
This Issue at a Glance: Skin Reactions of Astronauts in Space and Microstructures of Topically Applied Formulations
}

The analysis and characterization of the properties of human skin under natural conditions and under topical treatment on Earth is a topic of comprehensive investigation. In the present issue, it is demonstrated that there is also a skin physiology outside the Earth in the universe.

Tronnier et al. (Skin Pharmacol Physiol 2008;21:283-292) investigated the changes in skin physiological parameters in space. Astronauts often show skin reactions. In an interdisciplinary cooperation, a test series with skin physiological measurements was carried out before, during and after a long-term mission at the International Space Station. The hydration of the stratum corneum (SC), transepidermal water loss and the surface structure of the skin were measured. In order to record cutaneous states, suction elasticity was measured. Additionally, ultrasound measurements with $20 \mathrm{MHz}$ were made. There were indications of a delayed epidermal proliferation of cells, which would correspond to the clinical symptoms. Hydration and transepidermal water loss values are improved by respective skin care. At the cutaneous level, the elasticity measurements and the ultrasound picture showed results which correspond to a significant loss of elasticity of the skin.

The second main topic of this issue is the development and application of topically applied microstructured formulations.

Santos et al. (Skin Pharmacol Physiol 2008;21:246-259) reviewed the application of microemulsions in dermal and transdermal drug delivery. This review discusses the microemulsion nomenclature and composition, with particular emphasis on the choice of surfactant and cosurfactant. Methods used to characterize microemulsions are reviewed. Microemulsion formulations for dermal and transdermal delivery of pharmaceutical agents with particular emphasis on anti-inflammatory and anaesthetic agents are critically evaluated. Finally, issues that warrant further investigation by researchers in order to realize the full potential of the technology are discussed.

Lademann et al. (Skin Pharmacol Physiol 2008;21:274-282) investigated the influence of microparticles on the homogeneity of distribution of topically applied substances. In the study, the influence of microparticles on the homogeneity of distribution of a skin protection cream was investigated by laser scanning microscopy. The protection cream contained antioxidant substances with a high radical protection factor for the prevention of palmar-plantar erythema, often occurring during chemotherapy with doxorubicin. In this case, the chemotherapeutic substance comes out with the sweat onto the skin surface, from which it penetrates into the skin as if topically applied. lt was found that particles increase the homogeneity of distribution and, thus, the protection efficacy is significantly increased.

The fourth topic in this issue is related to the characterization and treatment of skin lesions.

Casas et al. (Skin Pharmacol Physiol 2008;21:260-268) characterized the inflammation and Staphylococcus aureus (SA) colonization of involved skin of atopic dermatitis (AD) patients at a molecular level. The aim of this work was to propose a new non-invasive approach to enumerate the genes coding for the toxins of SA in atopic skin samples. In parallel, the study aimed to evaluate the change in $\mathrm{AD}$ through three markers of inflammatory response: IL-8, IL-1RA/IL-1 $\alpha$ and IL-18. Finally, the authors found a positive correlation between increases in the Scoring Atopic Dermatitis Index after treatment and the corresponding evolution of SA density. These methods provide a means of clinically evaluating the course of $\mathrm{AD}$, and may help in the development of potential treatments.

Iwai and Hirao (Skin Pharmacol Physiol 2008;21:269-273) demonstrated in their study that protein carbonyls damage the water-holding capacity of the SC. Acrolein is a degradation product of lipid peroxide as well as a well-known environmental pollutant. As a hallmark of oxidatively damaged protein, protein carbonyl, including acrolein-protein adduct, has been observed in the skin.

Acrolein caused protein carbonylation, decreased the water-holding capacity and the bound water of the porcine SC in vitro. The water-holding capacity of the keratin gels prepared from human SC was also decreased by acrolein in vitro. Exposure of $\mathrm{SC}$ to the oxidative environment damages the waterholding capacity of the SC through the modification of protein-water interaction.

Jürgen Lademann, Editor Joachim Fluhr, President of the ISP

\section{KARGER}

Fax +41 613061234 E-Mail karger@karger.ch www.karger.com (c) 2008 S. Karger AG, Base

$1660-5527 / 08 / 0215-0245 \$ 24.50 / 0$

Accessible online at:

www.karger.com/spp 